\title{
Relations between genotype and phenotype in German patients with the Machado-Joseph disease mutation
}

Ludger Schöls, Georgios Amoiridis, Jörg T Epplen, Mathias Langkafel, Horst Przuntek, Olaf Riess

\begin{abstract}
Objective-Machado-Joseph disease (MJD) is an autosomal dominant cerebellar ataxia with extensive phenotypic variability originally described in families of Portuguese ancestry. Recently, the mutation causing the disease has been identified as an expanded CAG trinucleotide repeat. In this study relations between genotype and phenotype were investigated.
\end{abstract}

Methods-A series of 180 German patients with degenerative forms of ataxia were clinically and genetically examined. Patients bearing the MJD mutation were assigned to three phenotypes: phenotype 1 characterised by early onset and dystonia or pronounced rigidity associated with ataxia and spasticity. Main symptoms in phenotype 2 were ataxia and spasticity. In phenotype 3 onset was relatively late and peripheral neuropathy accompanied ataxia. Clinical and molecular data were correlated.

Results-An expanded CAG array was found in 42 patients from 22 families. Repeat length of CAG varied between 67 and 80 CAG motifs and showed an inverse correlation with the age of onset. For the development of phenotype 1 early onset ( $<20$ years) seemed more decisive than extensive repeat length. Phenotype 2 was present in all patients with more than 73 CAG motifs and onset between 20 and 40 . Phenotype 3 developed in most patients with less than 73 CAG motifs and onset was regularly beyond the age of 40 . Intrafamilial variability of both repeat length and phenotype was large reflecting meiotic instability of the expanded CAG repeat.

Conclusion-The MJD mutation is the most frequent cause of dominantly inherited ataxia in Germany. Variations in repeat lengths substantially influence age of onset as well as phenotype but cannot explain why MJD characteristics of Portuguese families such as "bulging eyes", dystonia, and rigidity are essentially missing in German families. Despite the genotypic and phenotypic relations found in this study a reliable individual prognosis of the course of the disease is not possible at a presymptomatic stage.

(F Neurol Neurosurg Psychiatry 1996;61:466-470)
Keywords: Machado-Joseph disease; spinocerebellar ataxia type 3; clinical features; molecular genetics

The spinocerebellar ataxias (SCAs) are a heterogeneous group of neurodegenerative disorders with a wide phenotypic variability. Recently, several genes causing genetically distinct forms of SCA have been mapped chromosomally. ${ }^{1-8}$ For spinocerebellar ataxia type 1 (SCA1) and Machado-Joseph disease (MJD) the mutations causing disease have been identified as expanded CAG triplet repeats. ${ }^{9}{ }^{10}$ CAG repeat expansions also cause spinobulbar muscular atrophy, ${ }^{11}$ Huntington's disease, ${ }^{12}$ and dentatorubral pallidoluysian atrophy (DRPLA). ${ }^{13}$ Inverse correlation of repeat length and age of onset has been shown for all of these disorders. ${ }^{14-18}$ Furthermore, the CAG repeat is unstable during transmission from parent to offspring with a tendency for expansion. ${ }^{19-24}$ The elongation of the CAG repeat in later generations results in earlier onset and a more severe course of the disease, a phenomenon known as anticipation. ${ }^{25-28}$ The differentiation of various subtypes of SCA at their genetic background gives an opportunity to redefine their clinical phenotypes and to study the relation between genotype and phenotype.

Machado-Joseph disease is an autosomal dominantly inherited spinocerebellar degeneration originally described in families of Portuguese ancestry. ${ }^{29-31}$ Pathological changes include neuronal loss and gliosis in the cerebellum (especially in the dentate nucleus but sparing the cerebellar cortex), the spinal cord (spinocerebellar tracts, Clark's columns, anterior horn cells, and the posterior columns, but not the corticospinal tract, although clinically pyramidal signs are frequent), the substantia nigra, red nucleus, subthalamic nucleus, cranial nerve nuclei, and peripheral nerves. ${ }^{32}$

The clinical presentation of MJD is very pleomorphic. Three subtypes have been described with the following characteristics ${ }^{33}$ : type 1 is characterised by pronounced pyramidal and extrapyramidal signs besides the common features of cerebellar ataxia and ophthalmoplegia. Type 1 shows earlier onset (20-30 years) and a more rapid course of the disease. The clinical presentation of type 2 is limited to cerebellar and pyramidal deficits without extrapyramidal signs. Age of onset in type 2 is intermediate (20-45 years). Type 3 is characterised by pronounced peripheral signs in addition to ataxia and shows later onset (40-60 years) with rather slow progression.

"Bulging eyes" due to lid retraction, dystonia, 
Figure 1 Correlation between age of onset, repeat length, and clinical phenotype in 42 patients of German ancestry with MFD/SCA3. A linear correlation between age of onset and number of $C A G$ motifs was obtained $(r=$ $-0.82, P<0.0001)$.

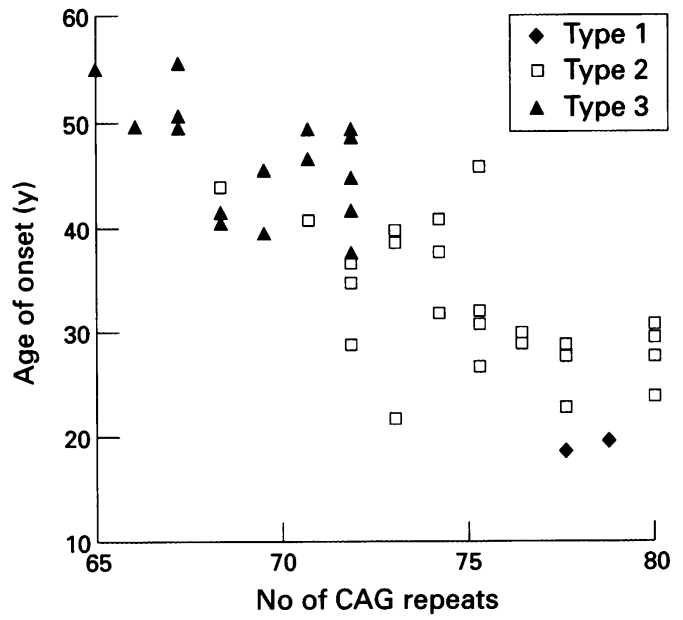

and faciolingual fasciculations are minor but rather characteristic features of $\mathrm{MJD} .^{34}$

German families with ataxia and bearing the MJD mutation present with a phenotype distinct from patients with MJD of Portuguese or Japanese ancestry ${ }^{35}$ but similar to French families described as spinocerebellar ataxia type 3 (SCA3). ${ }^{36}$ Recently, we proved that despite phenotypic differences both SCA3 and MJD are caused by mutations in the MFD1 gene. ${ }^{37}$

Here we present detailed clinical data for 42 patients with German ataxia bearing the MJD/SCA3 mutation and a study of the relation between the size of the expanded CAG repeat and the clinical presentation.

\section{Materials and methods}

A continuous series of 180 patients with degenerative forms of ataxia were clinically examined by one of us (LS) and blood samples were obtained for genetic analysis. The series included 77 patients from 45 families with a history of dominant inherited disease. All patients with autosomal dominant cerebellar ataxias (ADCAs) presented with symptoms such as cerebellar ataxia, external ophthalmoplegia, pyramidal and extrapyramidal features, polyneuropathy, and mild dementia consistent with ADCA type I according to the classification of Harding. ${ }^{38}$

Genomic DNA was extracted from peripheral white blood cells. ${ }^{39}$ Amplification of the CAG repeat by the polymerase chain reaction (PCR) was essentially carried out as described by Schöls et al..$^{37}$ Briefly, 100 ng genomic DNA was amplified by PCR using the primer sequences MJD52 and MJD25 ${ }^{10}$ in a total volume of $10 \mu \mathrm{l}$. Amplification was carried out for 30 cycles with denaturation at $95^{\circ} \mathrm{C}$ for one minute, annealing at $58^{\circ} \mathrm{C}$ for one minute, and extension at $72^{\circ} \mathrm{C}$ for one minute, in a Robocycler (Stratagene). Formamide was added to a final concentration of $1 \%$ to avoid non-specific amplification products. Products from PCR were electrophoresed in $6 \%$ denaturing polyacrylamide gels and sizes were determined by comparison with an M13 sequencing ladder. We included the AAG and two CAA variant triplets in determining the number of CAG repeats.
Statistical analysis of the relations between age of onset and CAG repeat number on the affected alleles was evaluated by linear regression analysis.

\section{Results}

An expanded CAG repeat in the MJD gene was found only in patients with a family history of dominant inheritance and not in patients with autosomal recessive or sporadic disease. From the $45 \mathrm{ADCA}$ kindreds included in this study five families with 11 patients had already been shown to have SCA1 based on the identification of the SCA1 mutation. ${ }^{14}$

The MJD mutation was found in 42 patients from 22 families with autosomal dominant cerebellar ataxia. All families were of German origin and no relation to Portuguese or Japanese ancestors could be found.

All 42 patients with MJD/SCA3 were heterozygous with one allele in the normal range (14-30 CAG motifs) and the other with an expanded trinucleotide repeat. Repeat lengths of the expanded alleles varied between 67 and 80 CAG motifs with 73 CAG repeats being the most often expanded allele size (eight patients).

Age of onset was inversely correlated with CAG repeat length $(r=-0.82, \mathrm{P}<0.0001$, fig 1). Patients with the same repeat length differed by up to 21 years in their age of onset (fig 1 , table 1). For example, in patients with $73 \mathrm{CAG}$ motifs onset varied between 29 and 50 years of age.

Characteristic signs of $\mathrm{MJD}$ as described in patients of Portuguese ancestry ${ }^{34}$ were not prominent in German patients bearing the MJD mutation. "Bulging eyes", rigidity, and dystonia were seen only in one of 42 patients. Faciolingual myokymia was present in a mild form in nine of 42 patients in our series, but similar facial movements were found in patients with ADCA not carrying the MJD mutation. Thus the German patients with the MJD genotype presented with the SCA3 phenotype. ${ }^{36}$

Initial complaints were problems with equilibrium and unsteadiness of gait in 38 of 42 patients with SCA3/MJD. Four patients reported diplopia as the first symptom, which preceded gait ataxia by up to 10 years. At the time of examination ataxia of gait, stance, and limbs were major clinical signs in all patients independent of repeat length (table 1). Cerebellar dysarthria (36 of 42) and cerebellar oculomotor signs such as gaze evoked nystagmus (41 of 42) and impaired smooth pursuit (38 of 42) were common.

As well as cerebellar dysfunction, peripheral neuropathy was a major clinical sign in most patients with relatively milder repeat expansions up to 72 CAG motifs (table 1). Muscle cramps and teasing dysesthaesiae were frequent complaints in these patients. Peripheral neuropathy signs included distally pronounced wasting of muscles in the feet and hands with mild paresis. Sensory loss was most pronounced in temperature discrimination followed by loss of vibration sense. Apart from two patients in whom pyramidal signs were more prominent than peripheral disorder all patients with less than 73 CAG 
Table 1 Correlation of $C A G$ repeat length and clinical features in 42 German patients bearing the MFD mutation

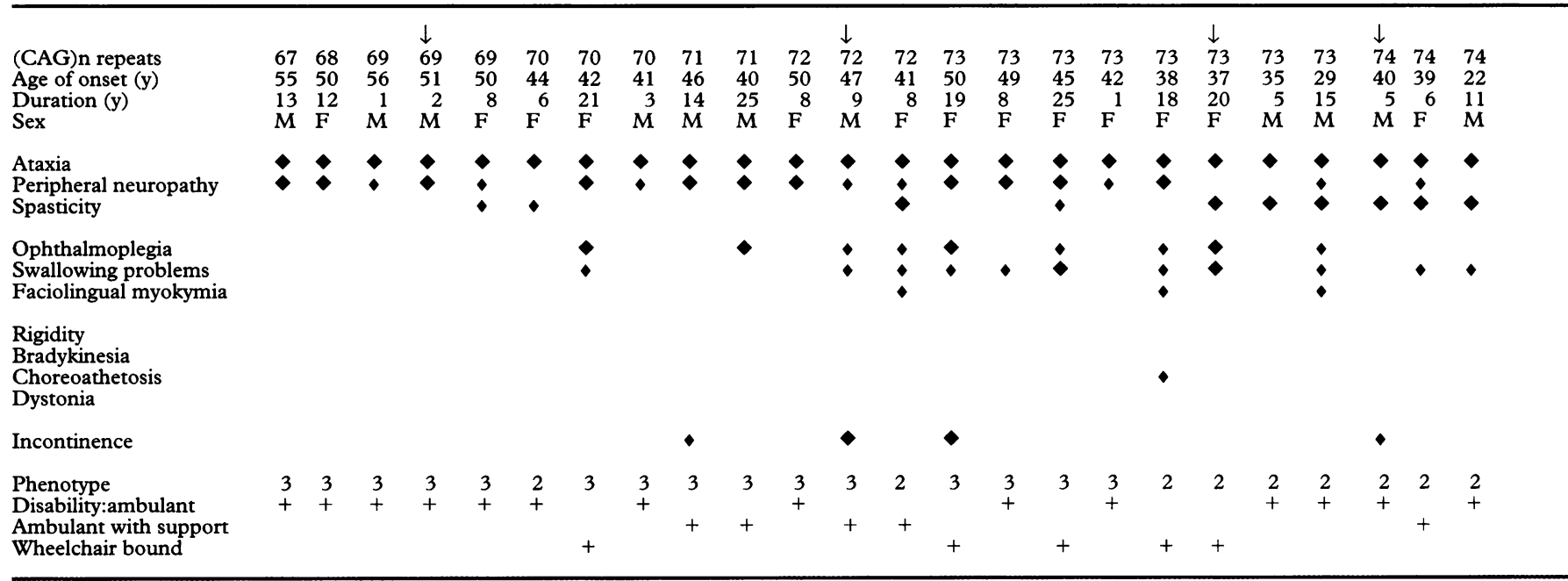

$\downarrow=$ major sign; $\downarrow=$ minor sign; $\downarrow=$ members of the SCA3/MJD kindred presented in fig 2.

repeats could be grouped as type 3 patients according to Coutinho and Andrade. ${ }^{33}$

Patients with 73 CAG motifs formed an intermediate group from their clinical appearance. The four patients with comparatively late onset $(50,49,45,42$ years) showed more peripheral signs, as type 3 patients and the four patients with earlier onset ( $38,37,35,29$ years) had more pronounced spasticity, as type 2 patients (see below)

In 21 patients exhibiting more than $73 \mathrm{CAG}$ motifs peripheral neuropathy was rarely a prominent problem (table 1 ). Instead spasticity evolved, especially in the legs (20 of 21). The gait was often characterised by stiffness (19 of 21). Hyperreflexia (16 of 21), extensor plantar responses (13 of 21), and spinal automatisms (11 of 21) were frequent signs. Due to the predominance of spasticity with ataxia we grouped these patients as type 2 after Coutinho and Andrade. ${ }^{33}$

Phenotype 1 (pronounced extrapyramidal features, especially rigidity and dystonia in combination with spasticity and ataxia) was seen in only two patients of our series. One patient had considerable rigidity combined with gross spasticity resulting in extreme stiffness which caused disablement of walking and most voluntary movements after 15 years of the disease. She reported that the clinical picture had changed during the disease. Unsteadiness of gait and clumsy hands had been the main problems during the first eight years, thereafter progressive stiffness became most disabling. The other type I patient was the only patient with significant dystonia in our series. After nine years of disease he developed abnormal posturing of the right arm during walking whereas his movements were inconspicuous (apart from ataxia) when he was sitting or standing. Dystonic movements were not found by the same neurologist after a disease duration of seven years.

The expanded alleles of these type 1 patients harboured 79 and 78 CAG repeats respectively. Remarkably, four patients with 80 CAG repeats did not show type 1 features despite their longer repeat expansions and durations of disease up to 10 years. They presented with classic type 2 phenotypes (table 1).

Extrapyramidal involvement was clinically apparent only in a few other patients. One had a mild form of rigidity not warranting the classification as type 1 . Two patients had bradykinesia but not as a prominent sign and without rigidity. Another patient presented with involuntary choreoathetotic movements of the fingers after a duration of disease of 18 years. Two patients, carrying 70 and 73 CAG repeats, developed myoclonus, especially of the proximal muscles of all limbs after 20 and 19 years of disease. Myoclonus was present in the relaxed patient as well as during voluntary movements. Both patients were already bedridden when myoclonus developed.

Bulbar and pseudobulbar signs, especially swallowing problems, were not serious in type 3 patients with repeat lengths of less than 72 CAG units. Only patients with a disease duration of more than 20 years developed mild problems in swallowing. By contrast, all patients with 72 or more CAG motifs (mostly type 2 and type 1 patients) developed dysphagia when the duration of the disease exceeded five years. Similarly, external ophthalmoplegia was less frequent in type 3 patients and was often associated with swallowing problems and longer duration (table 1).

Urge incontinence was a problem in eight of 42 patients without being correlated with repeat lengths, spasticity, or disease duration.

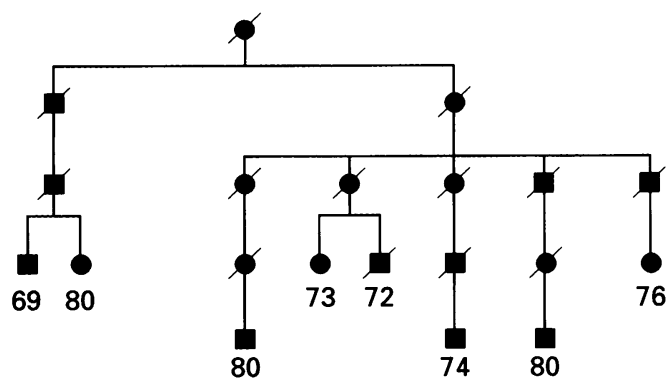

Figure 2 Family tree reduced to affected members of a large $S C A 3 / M F D$ kindred with wide variation of $C A G$ repeat expansions and clinical appearance (see table 1). 


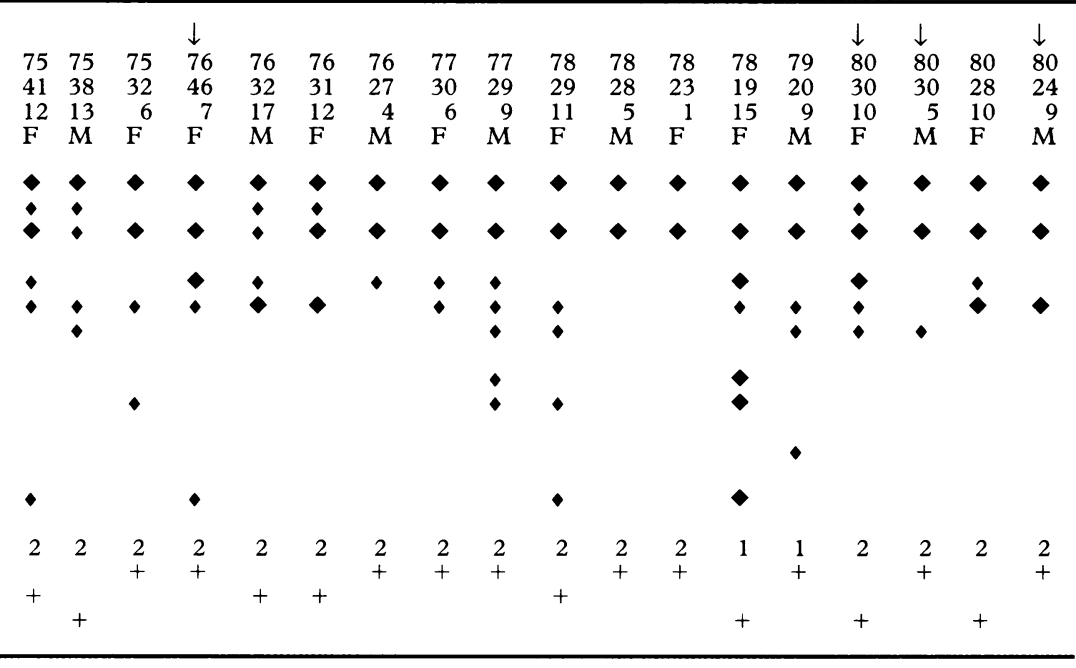

Table 2 Criteria for clinical subphenotypes in patients with SCA3/MFD according to Coutinho and Andrade ${ }^{23}$ with mild modifications as mentioned in the text

\begin{tabular}{lccc}
\hline & Type 1 & Type 2 & Type 3 \\
\hline Age of onset (y) & $10-30$ & $20-45$ & $40-60$ \\
Disease progression & Rapid & Intermediate & Slow \\
Major clinical features: & + to +++ & + to +++ & + to +++ \\
$\begin{array}{ll}\text { Cerebellar ataxia } \\
\text { Peripheral neuropathy }\end{array}$ & 0 to + & 0 to + & 0 \\
Pyramidal signs & ++ to +++ & ++ to +++ & 0 to +++ \\
EPS (rigidity, dystonia) & ++ to +++ & 0 to + & 0 to + \\
\hline $0=$ absent; $+=$ mild; $++=$ moderate; $+++=$ severe; ${ }^{\star}=$ at onset; EPS $=$ extrapyramidal
\end{tabular}
symptoms.
Molecular genetic data from the original publication $^{10}$ and further families of Portuguese and Japanese ancestry with classic MJD phenotypes $^{22}{ }^{23}$ did not differ from the repeat expansions in German families. Hence, the phenotypic differences of MJD and SCA3 remain unexplained at the molecular level but may be caused by the influence of other genes differing between patients with the MJD phenotype and those with the SCA3 phenotype. Haplotype analyses at flanking markers are conserved among Japanese and Azorean patients with MJD. ${ }^{23}$ Similar studies comparing Portuguese patients with the MJD phenotype and German patients with the SCA3 phenotype are under way to approach the question of common founders of the disease in these populations.

Despite clinical differences the criteria defining subphenotypes of MJD as elaborated by Coutinho and Andrade ${ }^{33}$ are well applicable to patients with the SCA3 phenotype, because clinical differences between SCA3 and MJD do not concern the criteria for subtypes apart from dystonia. However, we suggest a minor modification for the subclassification of patients with "pure" ataxia. Some of these patients develop more peripheral signs (type 3), others more pyramidal signs (type 2) later in the disease. In our experience noticeable spasticity develops, if at all, rather early (< five years) in the disease. Long standing "pure" ataxia is combined with rather slow progression and ends as type 3 . Therefore, we classified patients with SCA3/MJD with "pure" ataxia as type 3 patients and not as type 2 as recommended in the original report. ${ }^{32} 33$ Ophthalmoplegia does not seem to be helpful for the assignment of different subtypes in German patients with SCA3/MJD and was not strictly correlated with the expanded repeat length.

Some patients seem to start as type 2 and others as type 3 phenotypes. Some type 2 patients develop features of type 1 later in the disease and some type 3 patients change to intermediate type 2 or 3 appearance. This transitional form of disease was first described by Barbeau and coworkers. ${ }^{40}$ We never saw any patient with initial prominent spasticity (type 2) to develop a type 3 phenotype later on.

Using the subclassification recommended here (table 2) we found strong correlations between repeat lengths and subphenotypes in our series of patients with SCA3/MJD. Maruyama and coworkers ${ }^{24}$ did a similar study in Japanese patients with MJD using the original scheme. ${ }^{32}{ }^{33}$ In their study a substantial overlap existed concerning repeat lengths between type 2 and type 3 patients. The stronger correlation of repeat length and subtype in our study most likely results from a different grouping of ataxic patients without major peripheral or pyramidal signs as type 3 instead of type 2 as mentioned above.

This study confirms an inverse correlation between CAG repeat length and age of onset. According to our data the repeat length accounts for about $70 \%\left(r^{2}=0.67\right)$ of total variation in the age of onset. Besides the correlation of age of onset and repeat length it is 
important to recognise the influence of age of onset on clinical appearance. In our series this is seen best in the group of patients with 73 CAG repeats. Those with later onset ( $>40$ years) present as type 3 and those with earlier onset $(<40$ years) as type 2. Age of onset may be even more important for the development of subphenotype 1 as our type 1 patients had the earliest onset ( $<20$ years) but not the longest repeat expansions of our series. A similar tendency exists in the data of Maruyama et al..$^{24}$ However, the few type 1 patients in both studies preclude reliable evaluations.

Coexistence of two or more subphenotypes in one family has been reported for large MJD kindreds. ${ }^{41}{ }^{42}$ Here we present a family with SCA3/MJD with clinical variations warranting the classification in different subtypes even among sibs (type $2 v$ type 3; fig 2, table 1). Phenotypic variability also occurred in patients with similar repeat lengths as is seen for patients with 73 CAG repeats included in this study (table 1).

Therefore, a reliable individual prognosis of the course of the disease at a presymptomatic stage or in the beginning of the disease is not possible either on the basis of the repeat lengths or based on family history despite demonstrable genotype and phenotype correlations in general.

We thank Mrs AMM Vieira-Saecker for her excellent technical assistance. This study would not have been possible without the help of the SCA3/MJD families, who are gratefully acknowledged. This work was supported in part by grants from the self help group Deutsche Heredo-Ataxie Gesellschaft.

1 Yakura $\mathrm{H}$, Wakisaka A, Fujimoto S, Itakura K. Hereditary ataxia and HLA genotypes. $N$ Engl 7 Med 1974;291: $154-5$

2 Jackson JF, Currier RD, Terasaki PI, Morton NE. Spinocerebellar ataxia and HLA-linkage. Risk prediction by HLA typing. $N$ Engl F Med 1977;296:1138-41.

3 Gispert S, Twells R, Orozco G, et al. Chromosomal assignment of the second locus for autosomal dominant cerebellar ataxia (SCA2) to chromosome 12q23-24.1. Nature Genet 1993;4:295-9.

4 Takiyama Y, Nishizawa $M$, Tanaka $H$, et al. The gene for Machado-Joseph disease maps to human chromosome 14q. Nature Genet 1993;4:300-3.

5 Gardner K, Alderson K, Galster B, Kaplan C, Leppert M, Pracek L. Autosomal dominant spinocerebellar ataxia: clinical description of a distinct hereditary ataxia and genetic localization to chromosome 16 (SCA4) in a Utah kindred. Neurology 1994;44:A361.

6 Ranum LPW, Schut LJ, Lundgren JK, Orr HT, Livingston DM. Spinocerebellar ataxia type 5 in a family descended
from the grandparents of President Lincoln maps to chrofrom the grandparents of President Lincoln
mosome 11. Nature Genet 1994;8:280-4.

7 Benomar A, Krols L, Stevanin G, et al. The gene for autosomal dominant ataxia with pigmentary macular dystrophy maps to chromosome 3p12-p21.1. Nature Genet 1995;10: 84-8.

8 Gouw LG, Kaplan CD, Haines JH, et al. Retinal degeneration characterizes a spinocerebellar ataxia mapping to chromosome 3p. Nature Genet 1995;10:89-3.

9 Orr HT, Chung M, Banfi S, et al. Expansion of an unstable trinucleotide CAG repeat in spinocerebellar ataxia type 1. Nature Genet 1993;4:221-6.

10 Kawaguchi $Y$, Okamoto $T$, Taniwaki $M$, et al. CAG expansions in a novel gene for Machado-Joseph disease at chromosome 14q32.1. Nature Genet 1994;8:221-8.

$11 \mathrm{La}$ Spada AR, Wilson EM, Lubahn DB, Harding AE, Fischbeck $\mathrm{KH}$. Androgen receptor gene mutations in $\mathrm{X}$ linked spinal and bulbar muscular atrophy. Nature 1991; linked spinal

12 The Huntington's disease collaborative research group. A novel gene containing a trinucleotide repeat that is expanded and unstable on Huntington's disease chromosomes. Cell 1993;72:971-83.

13 Koide R, Ikeuchi T, Onodera O, et al. Unstable expansion of CAG repeat in hereditary dentatorubral-pallidoluysian atrophy (DRPLA). Nature Genet 1994;6:9-13.

14 Schöls L, Riess O, Schöls S, et al. Spinocerebellar ataxia type 1: clinical and neurophysiological characteristics in German kindreds. Acta Neurol Scand 1995;92:478-85.

15 Doyu M, Sobue G, Mukai E, et al. Severity of X-linked recessive bulbospinal neuronopathy correlates with size of the tandem CAG repeat in androgen receptor gene. Ann Neurol 1992;32:707-10.
16 Duyao M, Ambrose C, Myers R, et al. Trinucleotide repeat instability and age of onset in Huntington's disease. Nature Genet 1993;4:387-92.

17 Jodice C, Malaspina P, Persichetti F, et al. Effect of trinucleotide repeat length and parenteral sex on phenotypic variation in spinocerebellar ataxia 1. Am $\mathcal{f}$ Hum Genet 1993;2:2123-8.

18 Nagafuchi S, Yanagisawa $\mathrm{H}$, Sato $\mathrm{K}$, et al. Dentatorubral and pallidoluysian atrophy expansion of an unstable CAG trinucleotide on chromosome 12p. Nature Genet 1994;6: 14-8.

19 Dubourg O, Dürr A, Cancel G, et al. Analysis of the SCA1 CAG repeat in a large number of families with dominant ataxia: clinical and molecular correlations. Ann Neurol 1995;37:176-80.

20 Matilla T, McCall A, Subramony SH, Zoghbi HY. Molecular and clinical correlations in spinocerebellar ataxia type 3 and Machado-Joseph disease. Ann Neurol ataxia type 3 and

21 Komure O, Sano A, Nishino N, et al. DNA analysis in hereditary dentatorubral-pallidoluysian atrophy: Correlation between CAG repeat length and phenotypic variation and the molecular basis of anticipation. Neurology 1995; 45:143-9.

22 Maciel P, Gaspar C, De Stefano AL, et al. Correlation between CAG repeat length and clinical features in Machado-Joseph disease. Am f Hum Genet 1995;57:54-61.

23 Takiyama Y, Igarashi S, Rogaeva EA, et al. Evidence for inter-generational instability in the CAG repeat in the MJD1 gene and for conserved haplotypes at flanking markers amongst Japanese and Caucasian subjects with Machado-Joseph disease. Hum Mol Genet 1995;4: 1137-46.

24 Maruyama $H$, Nakamura S, Matsuyama $Z$, et al. Molecular features of the CAG repeats and clinical manifestation of Machado-Joseph disease. Hum Mol Genet 1995;4:807-12.

$25 \mathrm{La}$ Spada AR, Roling DB, Harding AE, et al. Meiotic stability and genotype-phenotype correlation of the trinucleotide repeat in X-linked spinal and bulbar muscular atrophy. Nature Genet 1992;2:301-4.

26 Andrew SE, Goldberg YP, Kremer B, et al. The relationship between trinucleotide (CAG) repeat length and clinical features of Huntington's disease. Nature Genet 1993;4: 398-403.

27 Ranum LPW, Chung M, Banfi S, et al. Molecular and clinical correlations in spinocerebellar ataxia type I: evidence for familial effects on the age of onset. Am $\mathcal{F}$ Hum Genet 1994;55:244-52.

28 Ikeuchi T, Koide R, Tanaka $\mathrm{H}$, et al. Dentatorubral-pallidoluysian atrophy: clinical features are closely related to unstable expansions of trinucleotide (CAG) repeat. Ann Neurol 1995;37:769-75.

29 Nakano KK, Dawson DM, Spence A. Machado disease: a hereditary ataxia in Portuguese immigrants to Massachusetts. Neurology 1972;22:49-55.

30 Woods BI, Schaumburg HH. Nigro-spino-dentatal degeneration with nuclear ophthalmoplegia: a unique and partially treatable clinicopathological entity. $\mathcal{F}$ Neurol Sci 1972;17: 149-66.

31 Rosenberg RN, Nyhan WL, Bay C, Shore P. Autosomal dominant striatonigral degeneration. A clinical, pathologic, and biochemical study of a new genetic disorder. Neurology 1976;26:703-14.

32 Sequeiros J, Coutinho P. Epidemiology and clinical aspects of Machado-Joseph disease. In: Harding AE, Deufel T, eds. Advances in neurology. Vol 61. New York: Raven Press, 1993;139-53.

33 Coutinho P, Andrade C. Autosomal dominant system degeneration in Portuguese families of the Azores Islands. A new genetic disorder involving cerebellar, pyramidal, extrapyramidal and spinal cord motor functions. Neurology 1978;28:703-9.

34 Lima L, Coutinho P. Clinical criteria for diagnosis of Machado-Joseph disease: report of a non-Azorean Portuguese family. Neurology 1980;30:319-22.

35 Schöls L, Amoiridis G, Langkafel M, et al. Machado-Joseph disease mutations as the genetic basis of most spinocerebellar ataxias in Germany. $\mathcal{F}$ Neurol Neurosurg Psychiatry 1995;59:449-50.

36 Stevanin G, Le Guern E, Ravise N, et al. A third locus for autosomal dominant cerebellar ataxia type I maps to chromosome 14q24-3-qter: evidence for the existence of a fourth locus. Am $\mathcal{F}$ Hum Genet 1994;54:11-20.

37 Schöls L, Vieira-Saecker AMM, Schöls S, Przuntek H, Epplen JT, Riess O. Trinucleotide expansion within the MJD1 gene presents clinically as spinocerebellar ataxia and occurs most frequently in German SCA patients. Hum and occurs most frequently in German SCA patients. Hum

38 Harding AE. The clinical features and classification of the late onset autosomal dominant cerebellar ataxias. Brain 1982;105:1-28.

39 Miller SA, Dykes DD, Polesky HF. A simple salting out procedure for extracting DNA from human nucleated cells. Nucleic Acids Res 1988;16:1215.

40 Barbeau A, Roy M, Cunha L, et al. The natural history of Machado-Joseph disease: an analysis of 148 personally examined cases. Can $\mathcal{F}$ Neurol Sci 1984;11:510-25.

41 Romanul FCA, Fowler HL, Radvany J, Feldman RG Feingold $M$. Azorean disease of the nervous system. $N$ Engl f Med 1977;296:1505-8.

42 Rosenberg RN. Machado-Joseph disease: an autosomal dominant motor system degeneration. Mov Disord 1992; 7:193-203. 